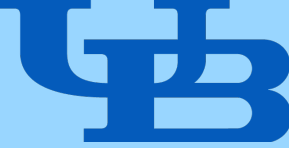

\title{
Simulation of CMS Phase 2 Pixel Tracker for HL-LHC
}

\author{
Bahareh Roozbahani
}

This manuscript has been authored by Fermi Research Alliance, LLC under Contract No. DE-AC02-07CH11359 with the U.S.

Department of Energy, Office of Science, Office of High Energy Physics.

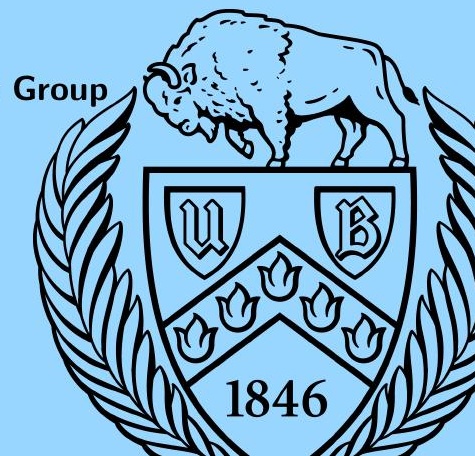




\section{Introduction}

- CMS pixel detector is a unique tracking detector

$\rightarrow$ All-silicon technology

$\rightarrow$ Key element in efficient and precise reconstruction of tracks/interaction vertices and heavy flavor tagging

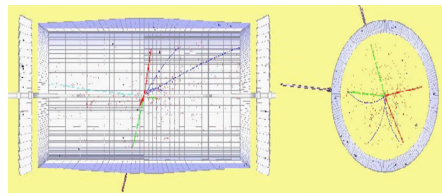

$$
\mathrm{H} \rightarrow \mathrm{ZZ} \rightarrow e e \mu \mu
$$

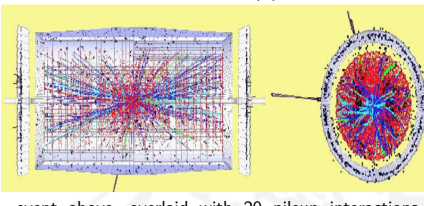
Difficult environment

event above, overlaid with 20 pileup interactions

$\rightarrow$ High instantaneous luminosity $\left(\sim 10^{34} \mathrm{~cm}^{-2} \mathrm{~s}^{-1}\right)$

$\rightarrow$ Large number of pp interactions per bunchcrossing (pileup)

$\rightarrow$ Expecting increase in instantaneous luminosity, pileup up to 140 to 200 at HL-LHC

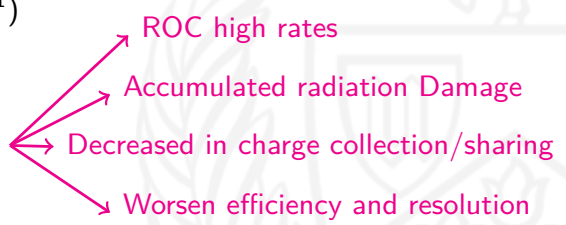




\section{CMS Pixel Detector Upgrade}

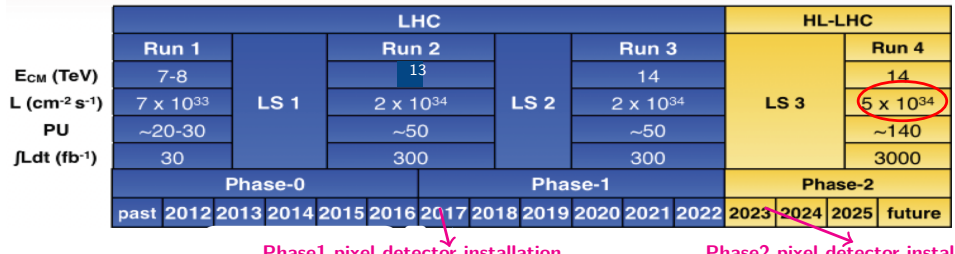

Phase1 pixel detector installation

Phase2 pixel detector installation

Phase $0 \rightarrow$ Phase1

- Added extra barrel layer and endcap disk

- Layers closer to the beam-line

$\rightarrow$ improvement in tracking and b-tagging

- Barrel: $48 \mathrm{M} \rightarrow 79 \mathrm{M}$ pixels

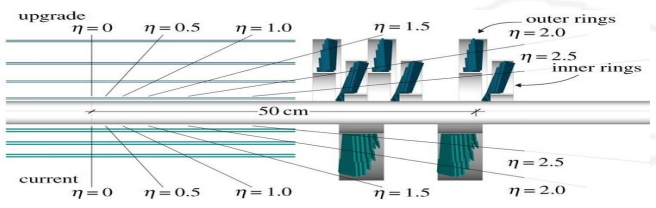

Forward: $18 \mathrm{M} \rightarrow 45 \mathrm{M}$ pixels

- Moved from analog to digital readout
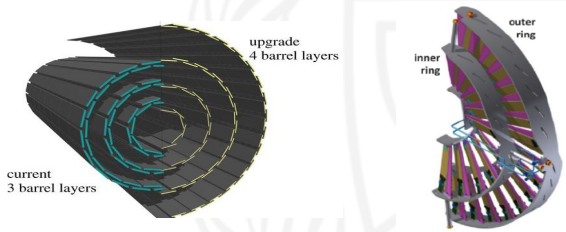


\section{CMS Pixel Detector Phase2 Upgrade}

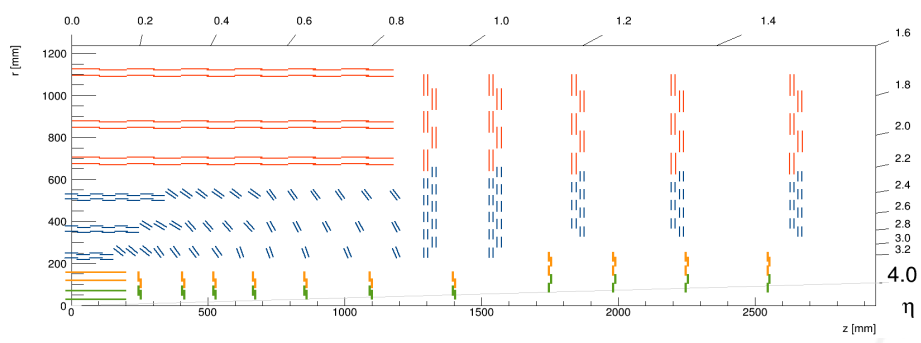

- Inner Tracker (Pixel Detector):

$\rightarrow$ Same number of Barrel layers (4) as the current detector

$\rightarrow$ Increase the endcap disks to 12 disks

Bette $\overline{\eta \text { coverage } \rightarrow|\eta|<4.0}$

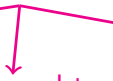

Improved tracking/vertexing

Better mitigation of pileup

$\rightarrow$ Increasing granularity/smaller pixels ( $\times 6$ smaller pixel area)

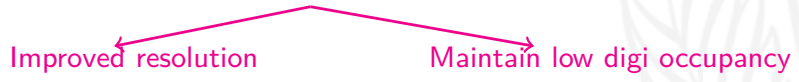




\section{Simulation and Digitization}

- 1) Simulation of the detector geometry using tkLayout

$\rightarrow$ geometry A: 4 barrel layer, 12 endcap disks

$$
25 \times 100 \times 150 \mu \mathrm{m}^{3} \text { pixels }
$$

$\rightarrow$ geometry B: 4 barrel layer, 12 endcap disks

$$
50 \times 50 \times 150 \mu \mathrm{m}^{3} \text { pixels }
$$

- 2) Simulation of desired physics processes using particle gun (Pythia8) and Detector response (Geant4)

$\rightarrow$ Ten Muon process with 200 pileup overlaid,

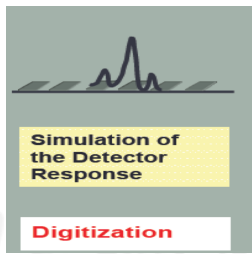
simulated in geometry A and geometry B

$\rightarrow$ Output is a collection of simulated hits

- 3) Digitization (CMSSW)

$\rightarrow$ Convert simulated hits to format similar to experimental raw data (digis)

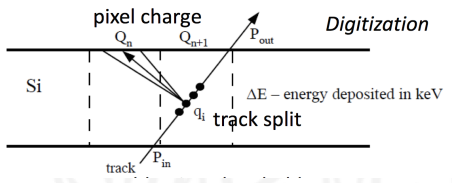




\section{Occupancy Study}
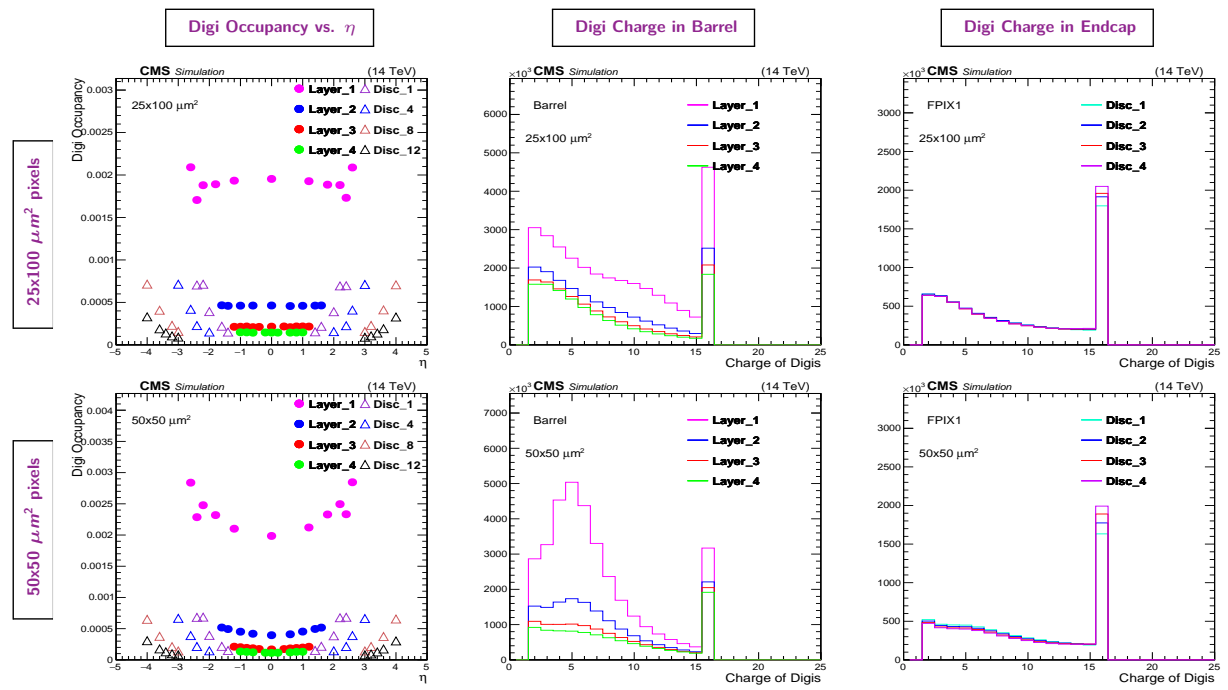

- Higher digi occupancy in the barrel for 50x50 $\mu \mathrm{m}^{2}$ comparing to $25 \times 100 \mu \mathrm{m}^{2}$

- Larger charge collection in $50 \times 50 \mu \mathrm{m}^{2}$ in the barrel, similar deposition in endcap 


\section{Track Reconstruction}

- step 1) Local Reconstruction

$\rightarrow$ Clustering adjacent pixel digis that are above certain threshold with 2 dimensional matrix algorithm
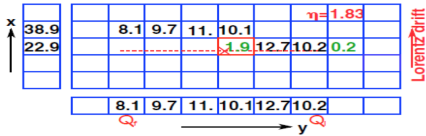

$\rightarrow$ Inputing clustered digis in a position estimator algorithm that take into account Lorentz drift to produce point measurements (RecHits)

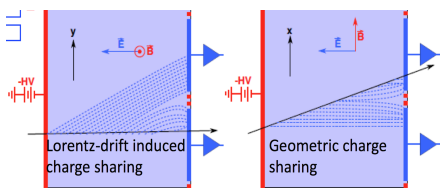

\section{- step 2) Track Reconstruction}

$\rightarrow$ Inputs are RecHits

$\rightarrow$ combinatory track finder (CTF) algorithm, combines reconstructed hits into tracks iteratively

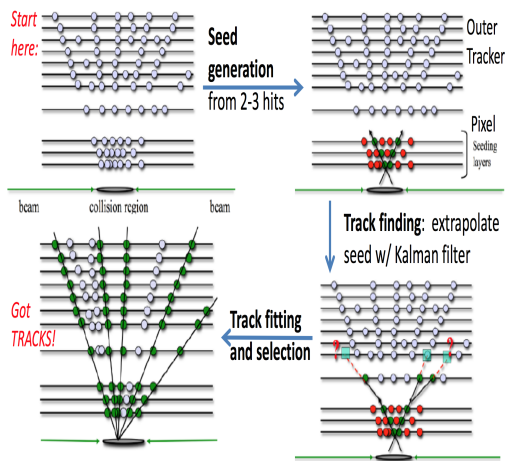




\section{Track transverse impact parameter $\left(d_{x y}\right)$ Resolution}
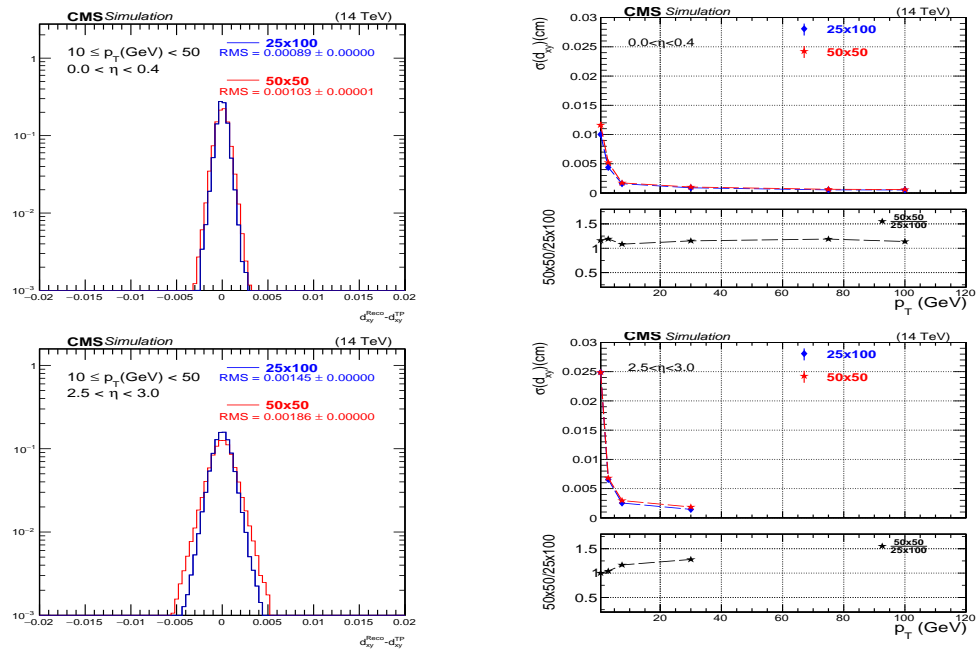

- $d_{x y}$ Resolution is worse in $50 \times 50 \mu \mathrm{m}^{2}$ geometry, particularly at high $p_{T}$ 


\section{Track longitudal impact parameter $\left(d_{z}\right)$ Resolution}
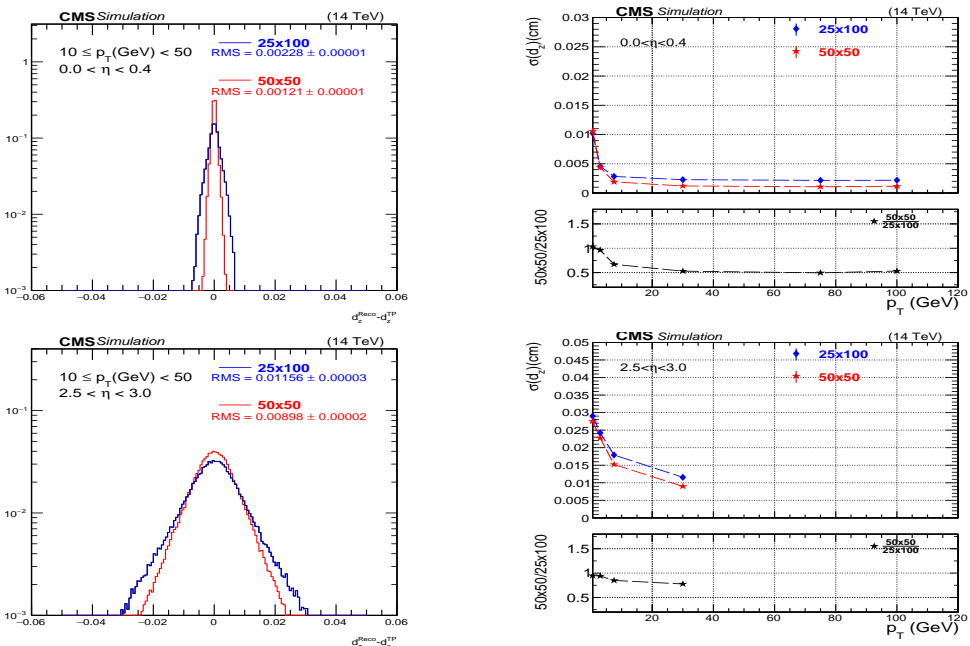

- $d_{z}$ Resolution is better in $50 \times 50 \mu m^{2}$ geometry, specially for higher $p_{T}$ tracking particles 


\section{Track $\boldsymbol{p}_{T}$ Resolution}
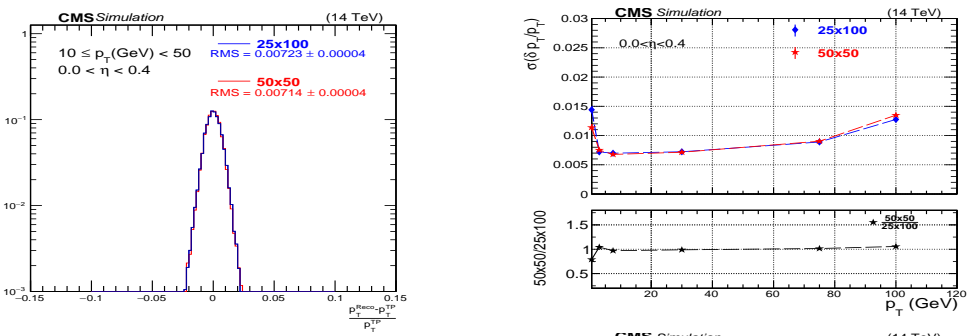

\begin{tabular}{|l|}
\hline+ \\
0 \\
$V$ \\
E \\
$V$ \\
0 \\
0 \\
\hline
\end{tabular}
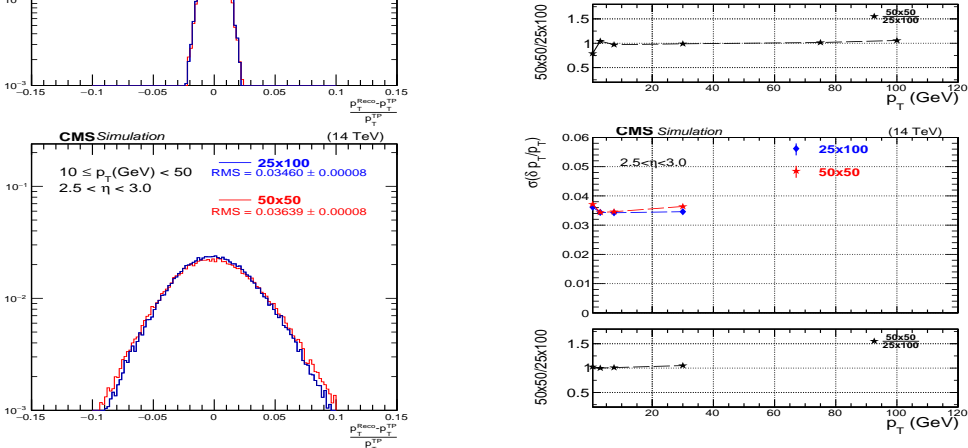

- $d_{p_{T}}$ Resolution is similar for $25 \times 100 \mu \mathrm{m}^{2}$ and $50 \times 50 \mu \mathrm{m}^{2}$ geometry, slightly worsen for higher $p_{T}$ in $50 \times 50 \mu \mathrm{m}^{2}$ 


\section{Summary}

- We have studied the pixel detector performance for 2 scenarios:

$\rightarrow 25 \times 100 \mu \mathrm{m}^{2}$ pixel size

$\rightarrow 50 \times 50 \mu \mathrm{m}^{2}$ pixel size

- Digi occupancy is somewhat higher in barrel for 50x50 $\mathrm{mm}^{2}$

- Larger charge collection in 50×50 $\mu^{2}$ comparing to $25 \times 100 \mu m^{2}$

- $d_{x y}$ resolutions are worse for $50 \times 50 \mu m^{2}$ in most $\eta$ bins

- $d_{z}$ resolutions are better for $50 \times 50 \mu \mathrm{m}^{2}$ in most $\eta$ bins

- $p_{T}$ resolutions are are similar for $50 \times 50 \mu \mathrm{m}^{2}$ and $25 \times 100 \mu \mathrm{m}^{2}$ at low $p_{T}$, but becomes worse for $50 \times 50 \mu m^{2}$ at $p_{T}>100 \mathrm{GeV}$. 


\section{Backup Slides}




\section{Digi Rates in Endcap (FPIX1: Disks 1-4)}
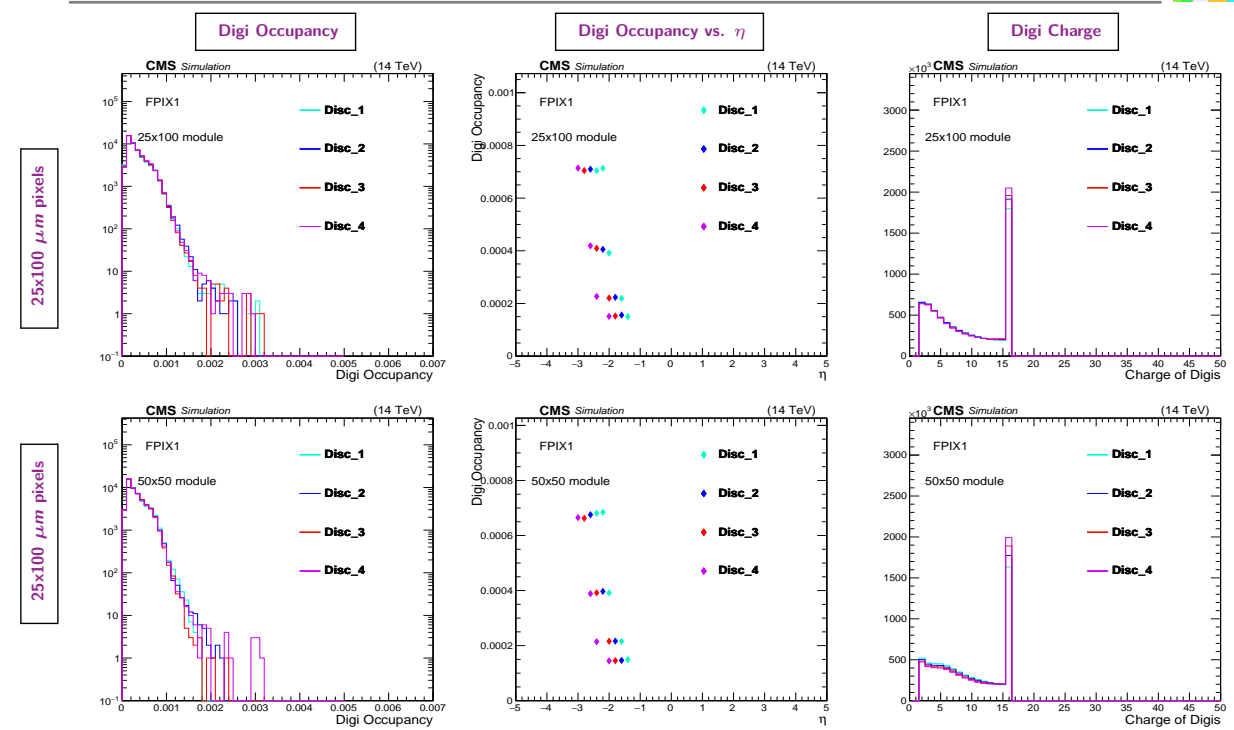


\section{Digi Rates in Endcap (FPIX1: Disks 1-4)}
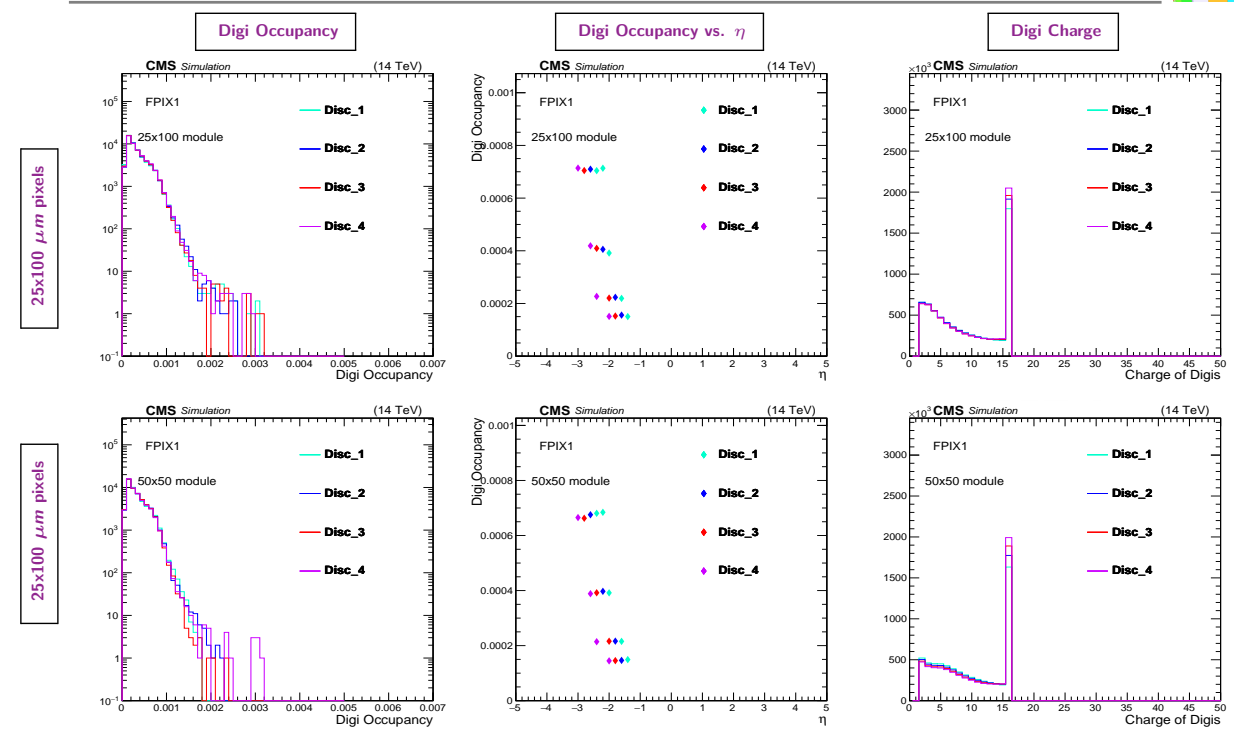


\section{Digi Rates in Endcap (FPIX2: Disks 1-4)}
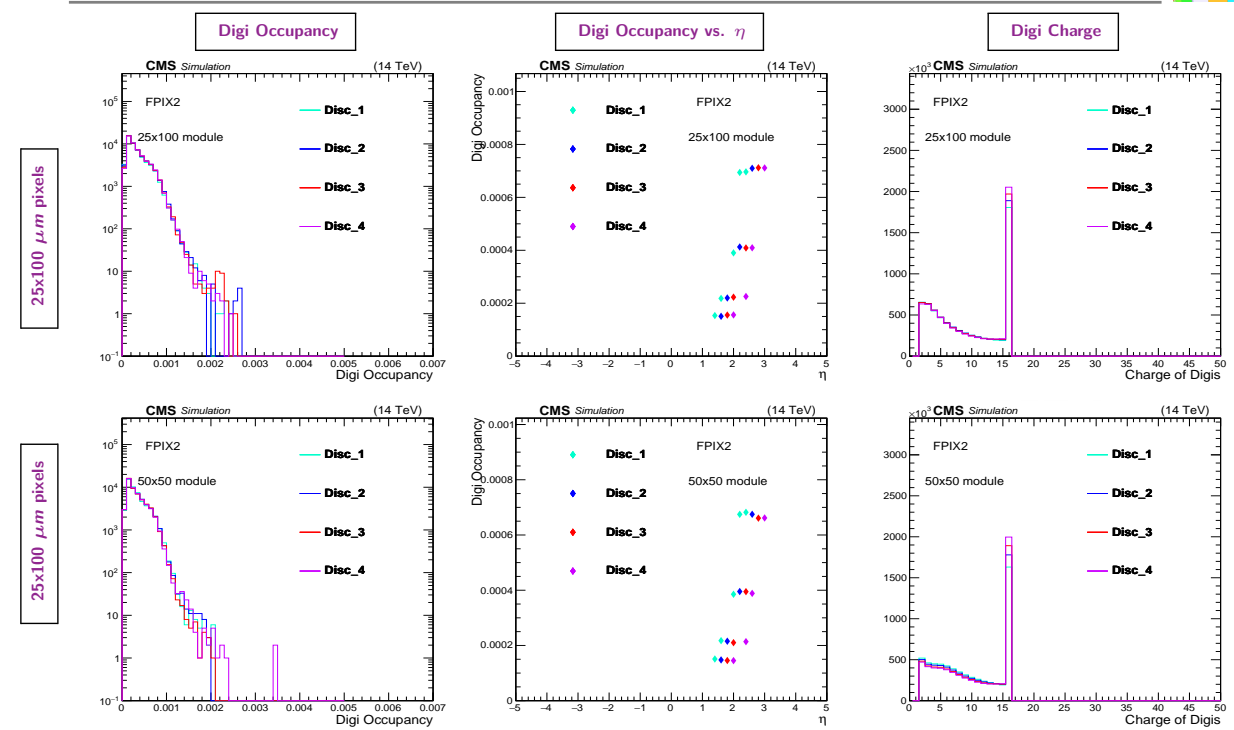

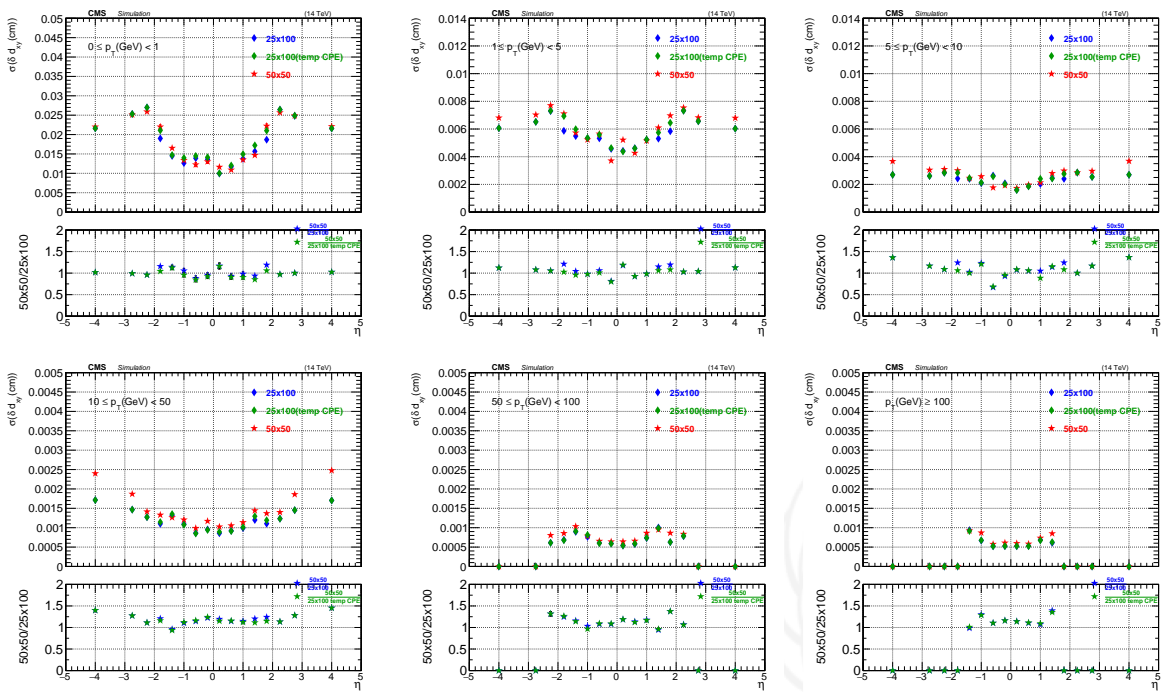

- $d_{x y}$ Resolution is worse in 50x50 geometry, particularly at high $p_{T}$.

- $d_{x y}$ Resolution is slightly worse for $25 \times 100$ with template CPEs comparing to $25 \times 100$ with generic CPEs. 

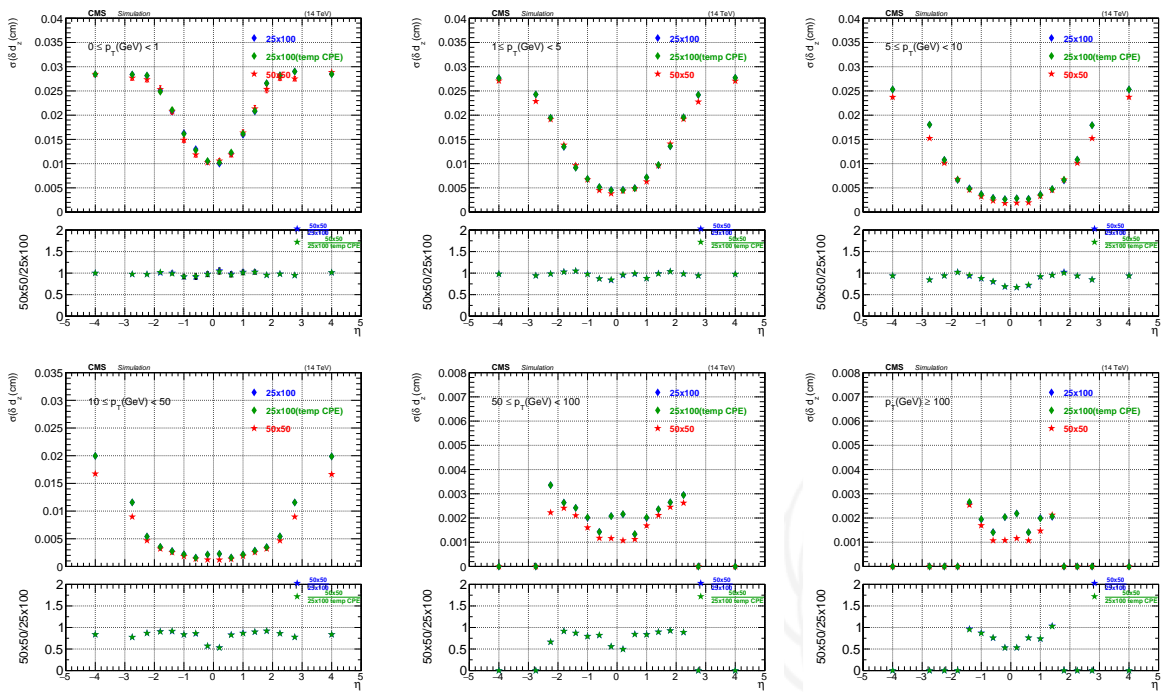

- $d_{z}$ Resolution is better in $50 \times 50$ geometry, specially for higher $p_{T}$ tracking particles.

- $d_{z}$ Resolution is similar for $25 \times 100$ with template CPEs comparing to $25 \times 100$ with generic CPEs. 